\title{
(ص) \\ Universality on higher order Hardy spaces
}

\author{
L. Bernal-González, A. Bonilla and M.C. Calderón-Moreno *
}

\begin{abstract}
We prove a Seidel-Walsh-type theorem about universality of a sequence of derivation-composition operators generated by automorphisms of the unit disk in the setting of the higher order Hardy spaces. Moreover, some related positive or negative assertions involving interpolating sequences and sequences between two tangent circles are established for the class of bounded functions in the unit disk. Our statements improve earlier ones due to Herzog and to the first and third authors.
\end{abstract}

2000 Mathematics Subject Classification: Primary 30E10. Secondary 30D55, 47A16, 47B38.

Key words and phrases: Unit disk, higher order Hardy spaces, SeidelWalsh theorem, bounded function, interpolating sequence, hypercyclic function, derivation-composition operator.

\section{Introduction and notation}

In this paper, we denote by $\mathbb{N}, \mathbb{C}, \mathbb{D}, \mathbb{N}_{0}$ the set of positive integers, the complex plane, the open unit disk $\{z \in \mathbb{C}:|z|<1\}$ and the set $\mathbb{N} \cup\{0\}$, respectively. The boundary of $\mathbb{D}$ is the unit circle $\partial \mathbb{D}=\{z \in \mathbb{C}:|z|=1\}$. If $G \subset \mathbb{C}$ is a domain (= nonempty connected open subset), then $H(G)$ stands for the Fréchet space of holomorphic functions on $G$ endowed with the topology of uniform convergence on compact subsets. A domain $G$ is said to be simply connected whenever its complement with respect to the extended plane is connected. The class $A(\mathbb{D})$ is the Banach space of all functions which are continuous on the closure $\overline{\mathbb{D}}$ of $\mathbb{D}$ and holomorphic in $\mathbb{D}$,

*The first and third authors have been partially supported by the Plan Andaluz de Investigación de la Junta de Andalucía FQM-127 and by DGES Grant BFM2003-03893-C02-01. The second author has been partially supported by MCYT-FEDER Project no. BFM 2002-02098. 
endowed with the supremum norm $\|\cdot\|_{\infty}$. If $1 \leq p<\infty$, the Hardy space $H^{p}(\mathbb{D})$ is defined as

$$
H^{p}(\mathbb{D})=\left\{f \in H(\mathbb{D}):\|f\|_{p}<\infty\right\},
$$

where $\|f\|_{p}=\sup _{r<1}\left(\frac{1}{2 \pi} \int_{0}^{2 \pi}\left|f\left(r e^{i \theta}\right)\right|^{p} d \theta\right)^{1 / p}$. Then $H^{p}(\mathbb{D})$ becomes a Banach space if it is endowed with this norm. And $H^{\infty}(\mathbb{D})$ is the space of all $f \in H(\mathbb{D})$ which are bounded on $\mathbb{D}$. It becomes a Banach space when endowed with $\|\cdot\|_{\infty}$. It is well known that for every $f \in H^{p}(\mathbb{D})$ the radial limit $f\left(e^{i \theta}\right)=\lim _{r \rightarrow 1} f\left(r e^{i \theta}\right)$ exists and is finite for almost all $\theta \in[0,2 \pi]$. In addition, $\|f\|_{p}=\left(\frac{1}{2 \pi} \int_{0}^{2 \pi}\left|f\left(e^{i \theta}\right)\right|^{p} d \theta\right)^{1 / p}$. See [5] for an extensive study of Hardy spaces.

For $N \in \mathbb{N}$, denote the higher order Hardy space

$$
H_{N}^{p}(\mathbb{D})=\left\{f \in H(\mathbb{D}): f^{(N)} \in H^{p}(\mathbb{D})\right\}
$$

which becomes a Banach space whenever it is endowed with the norm

$$
\|f\|=\left\|f^{(N)}\right\|_{p}+\sum_{j=0}^{N-1}\left\|f^{(j)}\right\|_{\infty} .
$$

For the sake of uniformity, the symbol $H_{0}^{p}(\mathbb{D})$ will denote $H^{p}(\mathbb{D})$. It is well known (see [5, Chapter 5, Exercise 9]) that if $f^{\prime} \in H^{p}(\mathbb{D})$ then $f \in A(\mathbb{D})$, so the norm $\|f\|$ above makes sense on $H_{N}^{p}(\mathbb{D})$. The polynomials are dense in $H_{N}^{p}(\mathbb{D})$. Higher order Hardy spaces are extensively studied in $[13]$. Note that $H_{N}^{p}(\mathbb{D}) \subset A^{N-1}(\mathbb{D}):=\{f \in$ $H(\mathbb{D}): f^{(j)} \in A(\mathbb{D})$ for $\left.j=0,1, \ldots, N-1\right\}$.

The group Aut $(\mathbb{D})$ of automorphisms of $\mathbb{D}$ is the set of Möbius transformations $\left\{\sigma_{a, k}:|a|<1=|k|\right\}$, where $\sigma_{a, k}(z)=k \cdot \frac{z-a}{1-\bar{a} z}$. In 1941 W. Seidel and J. L. Walsh [14] established the existence of a function $f \in H(\mathbb{D})$ such that, given a simply connected domain $G \subset \mathbb{D}$ and a function $g \in H(G)$, there is a sequence $\left\{a_{n}\right\}_{1}^{\infty} \subset \mathbb{D}$ depending on $g$ such that $f \circ \sigma_{a_{n}, 1} \rightarrow g(n \rightarrow \infty)$ in $H(G)$. This result is in turn a non-Euclidean version of Birkhoff's theorem about density of translates of certain entire functions [4]. Both results have been generalized and developed in several directions (see [9] for references), but in this paper we are mainly interested in a special one, namely, a version where composition-derivation make appearance. To this end, we need the next brief report.

In 1995, A. Montes-Rodríguez and the first author [3] extended Seidel-Walsh's theorem by showing that if $\left\{S_{n}=\sigma_{a_{n}, k_{n}}: n \in \mathbb{N}\right\} \subset \operatorname{Aut}(\mathbb{D})$, then the set $\{f \in$ $H(\mathbb{D}):\left\{f \circ S_{n}\right\}$ is dense in $\left.H(\mathbb{D})\right\}$ is not empty if and only if it is residual if and only if $\sup _{n \in \mathbb{D}}\left|a_{n}\right|=1$ if and only if the action of $\left\{S_{n}\right\}_{1}^{\infty}$ is properly discontinuous 
on $\mathbb{D}$, that is, given a compact subset $K \subset \mathbb{D}$ there exists $m=m(K) \in \mathbb{N}$ such that $K \cap S_{m}(K)=\emptyset$. In particular, if $\varphi=\sigma_{a, k}\left(|a|<1, k=e^{i \theta}\right)$ and $S_{n}=\varphi \circ \cdots \circ \varphi$ ( $n$ times), then the set $\left\{f \in H(\mathbb{D}):\left\{f \circ S_{n}\right\}\right.$ is dense in $\left.H(\mathbb{D})\right\}$ is not empty if and only if it is residual if and only if $\varphi$ has no fixed point in $\mathbb{D}$ if and only if $|\sin (\theta / 2)| \leq|a|$. In 1995, G. Herzog [11] proves the following "Seidel-Walsh theorem for derivatives": If $X$ is a Banach space of holomorphic functions on $\mathbb{D}$ with $A(\mathbb{D}) \subset X$ such that convergence in $X$ implies compact convergence on $\mathbb{D}$ and polynomials are dense in $X$, then for every sequence $\left\{a_{n}\right\} \subset \mathbb{D}$ with $\left|a_{n}\right| \rightarrow 1(n \rightarrow \infty)$ the set $\left\{f \in X:\left\{f^{\prime} \circ \sigma_{a_{n}, 1}: n \in \mathbb{N}\right\}\right.$ is dense in $\left.H(\mathbb{D})\right\}$ is a residual subset of $X$. Trivially, the expression $f^{\prime} \circ \sigma_{a_{n}, 1}$ cannot be changed to $f \circ \sigma_{a_{n}, 1}$ (just take $X=A(\mathbb{D})$ ). The assertion of Herzog's result is also obviously false for $X=A^{1}(\mathbb{D})$. In 1999, the first and third authors [2] extended Herzog's result to an operator of the form $\Phi(D)$, where $D$ is the differentiation operator $\left(D f=f^{\prime}\right)$ and $\Phi$ is a nonconstant polynomial, and in fact to a $C$-bounded sequence of polynomials. A sequence $\left\{\Phi_{n}(z)=\sum_{j=0}^{N} b_{j}^{(n)} z^{j}\right\}_{1}^{\infty}$ of polynomials of the same degree $N \in \mathbb{N}$ is $C$-bounded whenever each sequence $\left\{b_{j}^{(n)}: n \in \mathbb{N}\right\}(j=0,1,2, \ldots, N)$ is bounded and there exists a positive constant $\alpha$ such that $\left|b_{N}^{(n)}\right| \geq \alpha$ for all $n \in \mathbb{N}$. In [2] the following is shown: If $X$ is an $F$-space of holomorphic functions in $\mathbb{D}$ with $A(\mathbb{D}) \subset X$ such that convergence in $X$ implies compact convergence on $\mathbb{D}$ and polynomials are dense in $X$, and if $\left\{S_{n}\right\}_{1}^{\infty} \subset \operatorname{Aut}(\mathbb{D})$ and $\left\{\Phi_{n}\right\}_{1}^{\infty}$ is a $C$-bounded sequence of polynomials, then the set $\left\{f \in X:\left\{\Phi_{n}(D) \circ\right.\right.$ $\left.S_{n}: n \in \mathbb{N}\right\}$ is dense in $\left.H(\mathbb{D})\right\}$ is a residual subset of $X$ if and only if it is not empty if and only if the action of $\left\{S_{n}\right\}_{1}^{\infty}$ is properly discontinuous on $\mathbb{D}$.

All above density results can be expressed in the terminology of universality. If $X$ and $Y$ are topological vector spaces, then a sequence $T_{n}: X \rightarrow Y(n \in \mathbb{N})$ of continuous linear mappings is called universal or hypercyclic whenever the set $\mathcal{U}$ of elements $x \in X$ such that the orbit $\left\{T_{n} x\right\}_{1}^{\infty}$ is dense in $Y$ is not empty. Each element of $\mathcal{U}$ is said to be universal for $\left\{T_{n}\right\}_{1}^{\infty}$. See [9] and [10] for good updated surveys about these topics.

In this paper, we extend the main results of [2] and [11] to the higher order Hardy spaces $H_{N}^{p}(\mathbb{D})$, see Theorem 2.5 below. The conclusion is not true for $H^{\infty}(\mathbb{D})$. In fact, we will see in Section 3 that interpolating sequences and sequences lying on the region between two circles which are tangent to a boundary point do not generate "good" sequences of automorphisms in order to yield universality. This improves [11, Section 4].

\section{Universal functions in higher order Hardy spaces}

Before establishing the main result of this section, we need the four following statements, which can be found respectively in [8, Satz 1.2.2 and Satz 1.4.2] (see also [9, 
Proposition 6]) and [2, Lemmas 1, 2, 3].

Theorem 2.1. Let $X, Y$ be metrizable topological vector spaces with $X$ complete and $Y$ separable, and let $\Lambda=\left\{L_{n}\right\}_{1}^{\infty}$ be a sequence of continuous linear operators from $X$ to $Y$. Then the following statements are equivalent:

(a) The set of universal elements for $\Lambda$ is a residual subset of $X$.

(b) The set of universal elements for $\Lambda$ is a dense subset of $X$.

(c) The set $\left\{\left(x, L_{n}(x)\right): x \in X, n \in \mathbb{N}\right\}$ is dense in $X \times Y$.

If, in addition, there is a dense subset $C$ of $X$ such that $\lim _{n \rightarrow \infty} L_{n}(x)$ exists for all $x \in C$, then (a), (b) and (c) are equivalent to

(d) The set of universal elements for $\Lambda$ is not empty.

Lemma 2.2. Let $\left\{\Phi_{n}(z)=\sum_{j=0}^{N} a_{j}^{(n)} z^{j}\right\}_{n=1}^{\infty}$ be a sequence of polynomials with the same degree $N \in \mathbb{N}_{0}$ such that every sequence $\left\{a_{j}^{(n)}: n \in \mathbb{N}\right\}(j=0,1, \ldots, N)$ is bounded. Then there is a subsequence $\left\{\Phi_{n_{k}}: k \in \mathbb{N}\right\}$ and a polynomial $P$ satisfying that $\Phi_{n_{k}}(D) \varphi \rightarrow P(D) \varphi(k \rightarrow \infty)$ in $H(\mathbb{C})$ for every entire function $\varphi$.

Lemma 2.3. Assume that $G$ and $\Omega$ are two domains of $\mathbb{C}$ and that $H: \Omega \rightarrow \mathbb{C}$, $H_{k}: \Omega \rightarrow \mathbb{C}(k \in \mathbb{N}), \Psi: G \rightarrow \mathbb{C}, \Psi_{k}: G \rightarrow \mathbb{C}(k \in \mathbb{N})$ are functions satisfying the following properties:

(i) $H_{k}$ tends to $H(k \rightarrow \infty)$ uniformly on compacts sets in $\Omega$.

(ii) $\Psi_{k}$ tends to $\Psi(k \rightarrow \infty)$ uniformly on compacts sets in $G$.

(iii) $\Psi(G) \subset \Omega$.

(iv) $\Psi$ is continuous on $G$ and $H$ is continuous on $\Omega$.

Then $H_{k} \circ \Psi_{k} \rightarrow H \circ \Psi(k \rightarrow \infty)$ uniformly on compact subsets in $G$.

Lemma 2.4. Let $G \subset \mathbb{C}$ be a simply connected domain, $a \in G, F \in H(G)$ and, for each $k \in \mathbb{N}$,

$$
\left(I_{k} F\right)(z)=\int_{a}^{z} \frac{(z-\xi)^{k-1}}{(k-1) !} F(\xi) d \xi \quad(z \in G)
$$

where the integration is taken along any rectifiable curve in $G$ joining a to $z$. If we set $I_{0} F=F$, then $I_{k} F$ is well-defined for every $k \in\{0,1,2, \ldots\}, I_{k} F \in H(G)$ and $\left(I_{k} F\right)^{(j)}=I_{k-j} F$ for $j \in\{0,1, \ldots, k\}$. 
For the sake of completeness, we extend (with the same definition) the notion of $C$-boundedness to the case $N=0$, that is, when all $\Phi_{n}$ are constant.

Theorem 2.5. Assume that $N \in \mathbb{N}_{0}$. Let $X$ be an F-space of holomorphic functions on $\mathbb{D}$ having the following properties:

(a) Convergence in $X$ implies convergence on $\mathbb{D}$.

(b) $H_{N}^{p}(\mathbb{D}) \subset X$ for some $p \in[1, \infty)$.

(c) The polynomials are dense in $X$.

Assume that $\left\{S_{n}\right\}_{1}^{\infty}$ is a sequence of automorphisms of $\mathbb{D}$ and that $\left\{\Phi_{n}\right\}_{1}^{\infty}$ is a $C$ bounded sequence of polynomials of degree $N$. Denote $T_{n}=\Phi_{n}(D)(n \in \mathbb{N})$ and consider the set

$$
\mathcal{U}:=\left\{f \in X:\left\{\left(T_{n} f\right) \circ S_{n}: n \in \mathbb{N}\right\} \text { is dense in } H(\mathbb{D})\right\} .
$$

Then $\mathcal{U}$ is a residual set of $X$ if and only if $\mathcal{U}$ is not empty if and only if the action of $\left\{S_{n}\right\}_{1}^{\infty}$ is properly discontinuous on $\mathbb{D}$.

Proof. Assume that $N \in \mathbb{N}$. We have that $\Phi_{n}(z)=\sum_{j=0}^{N} b_{j}^{(n)} z^{j}(n \in \mathbb{N}),\left|b_{j}^{(n)}\right| \leq B_{j}<$ $+\infty(j=0,1, \ldots, N ; n \in \mathbb{N})$ and there exists $\alpha>0$ such that $\left|b_{N}^{(n)}\right| \geq \alpha(n \in \mathbb{N})$.

Each function $S_{n}$ has the form $S_{n}=\sigma_{a_{n}, k_{n}}$, where $\left|a_{n}\right|<1=\left|k_{n}\right|$ for all $n \in \mathbb{N}$. Suppose that the action of $\left\{S_{n}\right\}_{1}^{\infty}$ is not properly discontinuous on $\mathbb{D}$, so (see Section 1) $\lim \sup _{n \rightarrow \infty}\left|a_{n}\right|<1$. If $r \in(0,1)$ then (see the proof of Theorem 4 in [2]) we can associate it a number $c \in(0,1)$ such that $\left|S_{n}(z)\right| \leq c$ for all $n \in \mathbb{N}$. Given $f \in H(\mathbb{D})$, the set

$$
\bigcup_{n \in \mathbb{N}}\left[\left(\Phi_{n}(D) f\right) \circ S_{n}\right](\{|z| \leq r\})
$$

is bounded, because it is contained in the disk $\{|z| \leq s\}$ where $s:=$ $\sum_{j=0}^{N} B_{j} \sup _{|z| \leq c}\left|f^{(j)}(z)\right|<+\infty$. Thus, the set $\left\{\left(\Phi_{n}(D) f\right) \circ S_{n}: n \in \mathbb{N}\right\}$ cannot be dense in $H(\mathbb{D})$. Hence $\mathcal{U}$ is empty.

Now, the only property to be proved is that $\mathcal{U}$ is residual whenever $\sup _{n \in \mathbb{N}}\left|a_{n}\right|=1$. Let us prove it first in the case $X=H_{N}^{p}(\mathbb{D})$. Define the mappings

$$
L_{n}: H_{N}^{p}(\mathbb{D}) \rightarrow H(\mathbb{D}) \quad(n \in \mathbb{N})
$$


by $L_{n}(f)=\left(T_{n} f\right) \circ S_{n}$. Each $L_{n}$ is linear and continuous, because the fact $g_{j} \rightarrow 0$ $(j \rightarrow \infty)$ in $H_{N}^{p}(\mathbb{D})$ implies $g_{j}^{(\nu)} \rightarrow 0(j \rightarrow \infty)$ compactly in $\mathbb{D}(\nu=0,1, \ldots, N)$. If we prove that the set

$$
\mathcal{G}=\left\{\left(f, L_{n}(f)\right): f \in H_{N}^{p}(\mathbb{D}), n \in \mathbb{N}\right\}
$$

is dense in $H_{N}^{p}(\mathbb{D}) \times H(\mathbb{D})$ then an application of Theorem 2.1 would yield the conclusion in this case. Since the polynomials are dense in $H_{N}^{p}(\mathbb{D})$ and in $H(\mathbb{D})$, it is sufficient to prove that given a compact subset $K \subset \mathbb{D}$, two polynomials $p, q$ and $\varepsilon \in(0,1)$, there exist $g \in H_{N}^{p}(\mathbb{D})$ and $n_{0} \in \mathbb{N}$ such that

$$
\|p-g\|<\varepsilon
$$

and

$$
\left|q(z)-L_{n_{0}} g(z)\right|<\varepsilon \quad(z \in K)
$$

From the facts $\sup _{n \in \mathbb{N}}\left|a_{n}\right|=1$ and $\left|k_{n}\right|=1$ for every $n \in \mathbb{N}$, we can suppose with no loss of generality, by taking a subsequence if necessary, that there is a point $\gamma \in \partial \mathbb{D}$ such that $S_{n} \rightarrow \gamma(n \rightarrow \infty)$ uniformly on compact subsets of $\mathbb{D}$. Consider the function

$$
a(z)=\frac{1+\bar{\gamma} z}{2} \quad(z \in \mathbb{C}) .
$$

This is a "peak-function" at $\gamma$ for $\overline{\mathbb{D}}$ in the sense that $a(\gamma)=1$ and $|a(z)|<1$ for all $z \in \overline{\mathbb{D}} \backslash\{\gamma\}$ (see [6, page 189]).

Let $\beta=1+\|q\|_{\infty}+\sum_{j=0}^{N} B_{j}\left\|p^{(j)}\right\|_{\infty}$ and choose $m \in \mathbb{N}$ such that

$$
m>\frac{2 \beta\left(1+\sum_{j=0}^{N} B_{j}\right)(N+1)}{\alpha \varepsilon}
$$

and

$$
\left\|a^{m}\right\|_{p}<\frac{\alpha \varepsilon}{\beta(N+1)}
$$

The latter inequality is possible by the Lebesgue Bounded Convergence Theorem.

Moreover, since $S_{n} \rightarrow \gamma$ in $H(\mathbb{D})$ and $a(\gamma)=1$, there exists $n_{0} \in \mathbb{N}$ such that

$$
\sup _{z \in K}\left|1-\left[a\left(S_{n_{0}}(z)\right)\right]^{m}\right|<\frac{\varepsilon}{2 \beta} .
$$

Consider the function

$$
F(z)=\frac{a(z)^{m}}{b_{N}^{\left(n_{0}\right)}}\left[q\left(S_{n_{0}}^{-1}(z)\right)-\left(\Phi_{n_{0}}(D) p\right)(z)\right] .
$$


With the notation of Lemma 2.4, take $a=0$ and define the function

$$
h=I_{N} F
$$

on the domain $G=\left|a_{n_{0}}\right|^{-1} \mathbb{D}$. Then $h \in H\left(\left|a_{n_{0}}\right|^{-1} \mathbb{D}\right)$ and so $h \in H_{N}^{p}(\mathbb{D})$. If $z \in \overline{\mathbb{D}}$ and $j \in\{0,1, \ldots, N-1\}$ then we have

$$
\begin{gathered}
\left|h^{(j)}(z)\right|=\left|\frac{1}{b_{N}^{\left(n_{0}\right)}} \int_{0}^{z} \frac{(z-\xi)^{N-1-j}}{(N-1-j) !}\left(\frac{1+\bar{\gamma} \xi}{2}\right)^{m}\left[q\left(S_{n_{0}}^{-1}(\xi)\right)-\left(\Phi_{n_{0}}(D) p\right)(\xi)\right] d \xi\right| \\
=\left|\frac{1}{b_{N}^{\left(n_{0}\right)}} \int_{0}^{1} \frac{z^{N-1-j}(1-t)^{N-1-j} z}{(N-1-j) !}\left(\frac{1+\bar{\gamma} z t}{2}\right)^{m}\left[q\left(S_{n_{0}}^{-1}(z t)\right)-\left(\Phi_{n_{0}}(D) p\right)(z t)\right] d t\right| \\
<\frac{1}{\alpha} \int_{0}^{1} 1 \cdot \frac{(1+t)^{m}}{2^{m}} \cdot \beta d t=\frac{\beta}{\alpha 2^{m}(m+1)}\left(2^{m+1}-1\right) \\
<\frac{2 \beta}{\alpha m}<\frac{\varepsilon}{(N+1)\left(1+\sum_{j=0}^{N} B_{j}\right)},
\end{gathered}
$$

because of (1). Hence

$$
\left\|h^{(j)}\right\|_{\infty}<\frac{\varepsilon}{(N+1)\left(1+\sum_{j=0}^{N} B_{j}\right)} \quad \text { for } j \in\{0,1, \ldots, N-1\} .
$$

As for $h^{(N)}$, we obtain that

$$
\begin{gathered}
\left\|h^{(N)}\right\|_{p}=\|F\|_{p} \\
=\left(\frac{1}{2 \pi\left|b_{N}^{\left(n_{0}\right)}\right|^{p}} \int_{0}^{2 \pi}\left|a\left(e^{i \theta}\right)^{m}\right|^{p} \cdot\left|q\left(S_{n_{0}}^{-1}\left(e^{i \theta}\right)\right)-\left(\Phi_{n_{0}}(D) p\right)\left(e^{i \theta}\right)\right|^{p} d \theta\right)^{1 / p} \\
\leq \frac{\beta}{\alpha}\left\|a^{m}\right\|_{p}<\frac{\varepsilon}{N+1}
\end{gathered}
$$

by (2). Thus $\|h\|=\left\|h^{(N)}\right\|_{p}+\sum_{j=0}^{N-1}\left\|h^{(j)}\right\|_{\infty}<\varepsilon$.

Define $g=p+h$. Then $g \in H_{N}^{p}(\mathbb{D})$ and $\|g-p\|=\|h\|<\varepsilon$. Moreover,

$$
\begin{gathered}
q(z)-\left(L_{n_{0}} g\right)(z)=q(z)-\left(L_{n_{0}} p\right)(z)-\left(L_{n_{0}} h\right)(z) \\
=q(z)-\left(\Phi_{n_{0}}(D) p\right)\left(S_{n_{0}}(z)\right)-b_{N}^{\left(n_{0}\right)} h^{(N)}\left(S_{n_{0}}(z)\right)-\sum_{j=0}^{N-1} b_{j}^{\left(n_{0}\right)} h^{(j)}\left(S_{n_{0}}(z)\right) \\
=q(z)-\left(\Phi_{n_{0}}(D) p\right)\left(S_{n_{0}}(z)\right)-a\left(S_{n_{0}}(z)\right)^{m}\left[q(z)-\left(\Phi_{n_{0}}(D) p\right)\left(S_{n_{0}}(z)\right)\right]
\end{gathered}
$$




$$
\begin{gathered}
-\sum_{j=0}^{N-1} b_{j}^{\left(n_{0}\right)} h^{(j)}\left(S_{n_{0}}(z)\right) \\
=\left(1-a\left(S_{n_{0}}(z)\right)^{m}\right)\left(q(z)-\left(\Phi_{n_{0}}(D) p\right)\left(S_{n_{0}}(z)\right)\right)-\sum_{j=0}^{N-1} b_{j}^{\left(n_{0}\right)} h^{(j)}\left(S_{n_{0}}(z)\right)
\end{gathered}
$$

for all $z \in\left|a_{n_{0}}\right|^{-1} \mathbb{D}$. Now, if $z \in K$ then we get

$$
\begin{gathered}
\left|q(z)-\left(L_{n_{0}} g\right)(z)\right| \\
\leq\left|1-a\left(S_{n_{0}}(z)\right)^{m}\right| \cdot\left|q(z)-\left(\Phi_{n_{0}}(D) p\right)\left(S_{n_{0}}(z)\right)\right|+\sum_{j=0}^{N-1} B_{j}\left\|h^{(j)}\right\|_{\infty} \\
<\frac{\varepsilon}{2 \beta} \cdot \beta+\frac{\varepsilon}{2\left(1+\sum_{j=0}^{N} B_{j}\right)} \cdot \sum_{j=0}^{N-1} B_{j}<\varepsilon
\end{gathered}
$$

because of (3) and (4). Thus the closure of $\mathcal{G}$ contains the set $\{(p, q): p, q$ polynomials $\}$, which is dense in $H_{N}^{p}(\mathbb{D}) \times H(\mathbb{D})$, so $\mathcal{G}$ is also dense, as required.

Now, if $X$ is an $F$-space as in the hypothesis, then the mappings $L_{n}: X \rightarrow H(\mathbb{D})$ $(n \in \mathbb{N})$ are continuous by $(a)$. As before, we can assume that $\left\{S_{n}\right\}_{1}^{\infty}$ tends to a point $\gamma \in \partial \mathbb{D}$ in $H(\mathbb{D})$ and, by Lemma 2.2 , we may suppose with no loss of generality that there is a polynomial $P$ such that $T_{n} \varphi \rightarrow P(D) \varphi(n \rightarrow \infty)$ in $H(\mathbb{C})$ for every entire function $\varphi$. Fix a polynomial $\varphi$ and apply Lemma 2.3 on $G=\mathbb{D}, \Omega=\mathbb{C}, \Psi_{n}=S_{n}$, $\Psi=$ the constant $\gamma, H_{n}=T_{n} \varphi$ and $H=P(D) \varphi$. We obtain $L_{n} \varphi=\left(T_{n} \varphi\right) \circ S_{n} \rightarrow$ $(P(D) \varphi)(\gamma)(n \rightarrow \infty)$ in $H(\mathbb{D})$, so $\left(L_{n} \varphi\right)$ converges in $H(\mathbb{D})$ for every polynomial $\varphi$. Since (b) is satisfied, the set $\mathcal{U}$ is not empty. Finally, since $X$ satisfied $(c)$, an application of Theorem 2.1 with $Y=H(\mathbb{D})$ and $C=$ \{polynomials \} yields that $\mathcal{U}$ is a residual subset of $X$.

The remaining case $N=0$ is much easier and left to the reader. The proof is complete.

Note that the condition (b) in Theorem 2.5 is in some sense optimal, because the result is not true for $X \subset\left\{f \in H(\mathbb{D}): f^{(N)} \in H^{\infty}(\mathbb{D})\right\}$ (and, nevertheless, $\left.H_{N}^{p}(\mathbb{D}) \subset\left\{f \in H(\mathbb{D}): f^{(N-1)} \in A(\mathbb{D})\right\} \subset\left\{f \in H(\mathbb{D}): f^{(N-1)} \in H^{\infty}(\mathbb{D})\right\}\right)$. Indeed, if some member $f$ of $X$ were universal for $\left\{L_{n}\right\}_{1}^{\infty}$, where $L_{n} f=f^{(N)} \circ S_{n}$ (that is, we are taking $\Phi_{n}(z)=z^{N}$ for all $n$ ), we would get that $\left\{f^{(N)} \circ S_{n}: n \in \mathbb{N}\right\}$ is dense in $H(\mathbb{D})$, which is not possible since that set is bounded. 


\section{Bounded universal functions}

In $\left[11\right.$, Section 4] it is shown that there is a sequence $\left\{a_{n}\right\} \subset \mathbb{D}$ with $\lim _{n \rightarrow \infty}\left|a_{n}\right|=1$ such that the set $\left\{f \in H^{\infty}(\mathbb{D}):\left\{f^{\prime} \circ S_{n}: n \in \mathbb{N}\right\}\right.$ is dense in $\left.H(\mathbb{D})\right\}$ is not empty, but not dense in $H^{\infty}(\mathbb{D})$, where $S_{n}=\sigma_{a_{n}, 1}(n \in \mathbb{N})$. We furnish in this section an extension of this result into two directions.

Recall that a sequence $\left\{b_{n}\right\}_{1}^{\infty} \subset \mathbb{D}$ is said to be an interpolating sequence for $H^{\infty}(\mathbb{D})$ if and only if for every bounded sequence $\left\{c_{n}\right\}_{1}^{\infty}$ there exists $f \in H^{\infty}(\mathbb{D})$ such that $f\left(b_{n}\right)=c_{n}(n \in \mathbb{N})$ (see [5, Chapter 9], [6, Chapters 7 and 10], [12, Chapter $10]$ for a rather complete study of interpolating sequences). Note that $\left|b_{n}\right| \rightarrow 1$ $(n \rightarrow \infty)$ is a necessary condition for $\left\{b_{n}\right\}_{1}^{\infty}$ to be interpolating (in fact, $\sum\left(1-\left|b_{n}\right|\right.$ ) is convergent; see [5, page 150]).

Theorem 3.1. Assume that $\left\{S_{n}=\sigma_{a_{n}, k_{n}}\right\}_{1}^{\infty}\left(\left|a_{n}\right|<1=\left|k_{n}\right|\right.$ for all $\left.n \in \mathbb{N}\right)$ is a sequence of automorphisms of $\mathbb{D}$ such that $\left\{S_{n}(0)\right\}_{1}^{\infty}$ is an interpolating sequence. Then the set

$$
\mathcal{U}=\left\{f \in H^{\infty}(\mathbb{D}):\left\{f^{\prime} \circ S_{n}\right\}_{1}^{\infty} \text { is dense in } H(\mathbb{D})\right\}
$$

is nonempty, but not dense in $H^{\infty}(\mathbb{D})$.

Proof. Since $\left\{k_{n} a_{n}\right\}_{1}^{\infty}$ is interpolating then, as noted above, $\lim _{n \rightarrow \infty}\left|a_{n}\right|=1=$ $\lim _{n \rightarrow \infty}\left|k_{n} a_{n}\right|$, hence $\left\{S_{n}\right\}_{1}^{\infty}$ acts properly discontinuously on $\mathbb{D}$. Consequently, Theorem 3 in [11] (or our Theorem 2.5 with $N=1$ and $\Phi_{n}(z)=z$ for all $n \in \mathbb{N}$ ) guarantees that $\left\{f \in A(\mathbb{D}):\left\{f^{\prime} \circ S_{n}\right\}_{1}^{\infty}\right.$ is dense in $\left.H(\mathbb{D})\right\}$ is nonempty, whence $\mathcal{U}$ is also nonempty because $A(\mathbb{D}) \subset H^{\infty}(\mathbb{D})$.

Let us show that $\mathcal{U}$ is not dense in $H^{\infty}(\mathbb{D})$. Denote $b_{n}=-k_{n} a_{n}(n \in \mathbb{N})$. It is trivial that $\left\{b_{n}\right\}_{1}^{\infty}$ is also an interpolating sequence. Consider the Blaschke product

$$
B(z)=\prod_{1}^{\infty} \frac{\left|b_{n}\right|}{b_{n}} \cdot \frac{b_{n}-z}{1-\overline{b_{n}} z},
$$

where the $n$th factor should be changed to $z$ if $b_{n}=0$. The condition $\sum\left(1-\left|b_{n}\right|\right)<$ $+\infty$ guarantees the normal convergence of the infinite product on each compact subset of $\mathbb{D}$, so $B \in H^{\infty}(\mathbb{D})$. A straightforward calculation shows that

$$
\left|B^{\prime}\left(b_{n}\right)\right|=\frac{1}{1-\left|b_{n}\right|^{2}} \prod_{j \neq n}\left|\frac{b_{j}-b_{n}}{1-b_{j} \overline{b_{n}}}\right| .
$$

By Carleson's theorem [6, pages 284-294], the sequence $\left\{b_{n}\right\}_{1}^{\infty}$ is uniformly separated, that is, there is a constant $\varepsilon>0$ such that

$$
\prod_{j \neq n}\left|\frac{b_{j}-b_{n}}{1-b_{j} \overline{b_{n}}}\right| \geq \varepsilon \quad(n \in \mathbb{N}) .
$$


Thus,

$$
\left(1-\left|b_{n}\right|^{2}\right)\left|B^{\prime}\left(b_{n}\right)\right| \geq \varepsilon \quad(n \in \mathbb{N}) .
$$

By the way of contradiction, assume that $\mathcal{U}$ is dense. Then the set $\left\{\left(f, L_{n} f\right): f \in\right.$ $\left.H^{\infty}(\mathbb{D}), n \in \mathbb{N}\right\}$ is dense in $H^{\infty}(\mathbb{D}) \times H(\mathbb{D})$ by Theorem 2.1 , where we have set $L_{n} f=f^{\prime} \circ S_{n}$. In particular, there should exist a function $h \in H^{\infty}(\mathbb{D})$ and $m \in \mathbb{N}$ such that

$$
\|B-h\|_{\infty}<\frac{\varepsilon}{3}
$$

and

$$
\left|\left(h^{\prime} \circ S_{m}\right)(0)\right|=\left|h^{\prime}\left(b_{m}\right)\right|<\frac{\varepsilon}{3},
$$

because $\{0\}$ is a compact subset of $\mathbb{D}$ and the null function is trivially in $H(\mathbb{D})$. By using the well-known inequality

$$
\left(1-|z|^{2}\right)\left|\varphi^{\prime}(z)\right| \leq\|\varphi\|_{\infty} \quad\left(\varphi \in H^{\infty}(\mathbb{D})\right),
$$

we obtain that

$$
\begin{aligned}
& \frac{\varepsilon}{3}>\left|h^{\prime}\left(b_{m}\right)\right| \geq\left|B^{\prime}\left(b_{m}\right)\right|-\left|B^{\prime}\left(b_{m}\right)-h^{\prime}\left(b_{m}\right)\right| \\
& \geq \frac{\varepsilon}{1-\left|b_{m}\right|^{2}}-\frac{\|B-h\|_{\infty}}{1-\left|b_{m}\right|^{2}}>\frac{\varepsilon-(\varepsilon / 3)}{1-\left|b_{m}\right|^{2}} \geq \frac{2 \varepsilon}{3},
\end{aligned}
$$

which is a contradiction.

The last theorem proves that condition (c) in Theorem 2.5 cannot be omitted in general. Moreover, we remark that given a closed subset $E \subset \partial \mathbb{D}$ there exists $\left\{S_{n}\right\}_{1}^{\infty} \subset$ Aut $(\mathbb{D})$ such that the cluster set of $\left\{S_{n}(0)\right\}_{1}^{\infty}$ is exactly $E$ and the corresponding set $\mathcal{U}$ is not dense in $H^{\infty}(\mathbb{D})$. Indeed, Proposition 1.2 of [7] shows the existence of an interpolating sequence in $\mathbb{D}$ whose cluster set is a prefixed closed set $E \subset \partial \mathbb{D}$.

The following notion can be found in [1] (see also [9]). Given two topological vector spaces $X, Y$ and a sequence $T_{n}: X \rightarrow Y(n \in \mathbb{N})$ of continuous mappings, then $\left\{T_{n}\right\}_{1}^{\infty}$ is said to be densely hereditarily hypercyclic whenever the set $\left\{x \in X:\left\{T_{n_{k}} x: k \in\right.\right.$ $\mathbb{N}\}$ is dense in $Y\}$ is dense in $X$ for every sequence $\left\{n_{1}<n_{2}<\cdots<n_{k}<\cdots\right\}$ of positive integers.

Corollary 3.2. Define $T_{n}: f \in H^{\infty}(\mathbb{D}) \mapsto f^{\prime} \circ S_{n} \in H(\mathbb{D})$, where $\left\{S_{n}\right\}_{1}^{\infty} \subset \operatorname{Aut}(\mathbb{D})$. Then $\left\{T_{n}\right\}_{1}^{\infty}$ is not densely hereditarily hypercyclic.

Proof. We can write $S_{n}=\sigma_{a_{n}, k_{n}}$, where $\left|a_{n}\right|<1=\left|k_{n}\right|(n \in \mathbb{N})$. If $\lim _{\sup } \sup _{n \rightarrow \infty}\left|a_{n}\right|<$ 1 then, given a compact subset $K \subset \mathbb{D}$, the set $K_{1}:=\bigcup_{n \in \mathbb{N}} S_{n}(K)$ is relatively compact in $\mathbb{D}$. So, if $f \in H(\mathbb{D})$ then $\left|f^{\prime}(w)\right| \leq M<+\infty\left(w \in K_{1}\right)$ for some constant $M$. Therefore, $\left\{f^{\prime} \circ S_{n}\right\}_{1}^{\infty}$ cannot approximate the constant function $1+M$ on $K$. 
This shows that $\left\{T_{n}\right\}_{1}^{\infty}$ is not hypercyclic, so it is not densely hereditarily hypercyclic in this case.

If $\lim \sup _{n \rightarrow \infty}\left|a_{n}\right|=1$ then $\lim \sup _{n \rightarrow \infty}\left|k_{n} a_{n}\right|=1$ too and there is a subsequence $\left\{k_{n(j)} a_{n(j)}\right\}_{1}^{\infty}$ that is an interpolating sequence (see [12, page 204]). Then Theorem 3.1 applies and it is obtained that $\left\{f \in H^{\infty}(\mathbb{D}):\left\{T_{n(j)} f\right\}_{1}^{\infty}\right.$ is dense in $\left.H(\mathbb{D})\right\}$ is not dense in $H^{\infty}(\mathbb{D})$. The proof is complete.

The same negative conclusion of Theorem 3.1 can be achieved if the sequence $\left\{k_{n} a_{n}\right\}_{1}^{\infty}$ is in the region determined by two circles which are tangent to a boundary point.

Theorem 3.3. Assume that $\left\{S_{n}=\sigma_{a_{n}, k_{n}}\right\}_{1}^{\infty}\left(\left|a_{n}\right|<1=\left|k_{n}\right|\right.$ for all $\left.n \in \mathbb{N}\right)$ is a sequence of automorphisms of $\mathbb{D}$ such that there exist two points $a, b \in \mathbb{D} \backslash\{0\}$ on the same radius from the origin with $|a|<|b|$ and

$$
\left\{k_{n} a_{n}: n \in \mathbb{N}\right\} \subset\{z:|z-a|<1-|a| \text { and }|z-b|>1-|b|\} .
$$

Then the set

$$
\mathcal{U}:=\left\{f \in H^{\infty}(\mathbb{D}):\left\{f^{\prime} \circ S_{n}\right\}_{1}^{\infty} \text { is dense in } H(\mathbb{D})\right\}
$$

is not dense in $H^{\infty}(\mathbb{D})$.

Proof. Let us denote $G:=\{z:|z-a|<1-|a|$ and $|z-b|>1-|b|\}$. With no loss of generality, it can be assumed that the radius is $(-1,0)$, in such a way that $-1<b<a<0$, i.e. the circles of $\partial G$ are tangent to -1 . Then $\left\{b_{n}\right\}_{1}^{\infty} \subset G_{1}:=-G=$ $\{z:|z-c|<1-c$ and $|z-d|>1-d\}$ where $c=-a, d=-b$ and $b_{n}=-k_{n} a_{n}$ $(n \in \mathbb{N})$.

The point of the proof is to find a bounded holomorphic function $f$ such that circles in $\mathbb{D}$ which are tangent to 1 are level curves for the function $\left(1-|z|^{2}\right)\left|f^{\prime}(z)\right|$.

Consider the function $f(z)=\exp \left(\frac{z+1}{z-1}\right)$. We have that $z \in \mathbb{D}$ if and only if $\operatorname{Re}\left(\frac{z+1}{z-1}\right)<0$, so $f \in H^{\infty}(\mathbb{D})$. A simple computation gives us $\left(1-|z|^{2}\right)\left|f^{\prime}(z)\right|=$ $\frac{2\left(1-|z|^{2}\right)}{|z-1|^{2}} \exp \left(\operatorname{Re}\left(\frac{z+1}{z-1}\right)\right)$. On the other hand, given $\delta>0$, a little calculation shows that $\left(1-|z|^{2}\right)>\delta|z-1|^{2}$ if and only if $|z-\mu|<1-\mu$, where $\mu=\frac{\delta}{1+\delta} \in(0,1)$. Analogously, given $\alpha<0$, it happens that $\operatorname{Re}\left(\frac{z+1}{z-1}\right)>\alpha$ if and only if $|z-\nu|>1-\nu$, where $\nu=\frac{1}{1-\alpha} \in(0,1)$. Therefore $\left(1-|z|^{2}\right)\left|f^{\prime}(z)\right|>\varepsilon$ for all $z \in G_{1}$, where $\varepsilon:=\frac{2 c}{1-c} \exp \left(\frac{d-1}{d}\right)>0$. In particular,

$$
\left(1-\left|b_{n}\right|^{2}\right)\left|f^{\prime}\left(b_{n}\right)\right|>\varepsilon \quad(n \in \mathbb{N}) .
$$

From now on, one can follow step by step the final part of the proof of Theorem 3.1 just by changing $B$ to $f$. The proof is complete. 
To finish, we want to pose the following question, which arises naturally from the results of this section (see also [11, Section 5]): Are there sequences $\left\{S_{n}\right\}_{1}^{\infty} \subset$ $\operatorname{Aut}(\mathbb{D})$ such that $\left\{f \in H^{\infty}(\mathbb{D}):\left\{f^{\prime} \circ S_{n}\right\}_{1}^{\infty}\right.$ is dense in $\left.H(\mathbb{D})\right\}$ is dense in $H(\mathbb{D})$ ? Take into account that if $\left\{a_{n}\right\}_{1}^{\infty}$ is a separated sequence (or it contains a separated subsequence) such that the corresponding measure $\sum_{1}^{\infty}\left(1-\left|a_{n}\right|\right) \delta_{a_{n}}$ is not a Carleson measure (see [6] for definitions) then there is not a function $f \in H^{\infty}(\mathbb{D})$ satisfying that $\inf _{n}\left(1-\left|a_{n}\right|^{2}\right)\left|f^{\prime}\left(a_{n}\right)\right|>\varepsilon$ for some $\varepsilon>0$. Thus, it is not possible to do a reasoning analogous to those in Theorems 3.1 and 3.3.

\section{References}

[1] L. Bernal-González, Densely hereditarily hypercyclic sequences and large hypercyclic manifolds, Proc. Amer. Math. Soc. 127 (1999), 3279-3285.

[2] L. Bernal-González and M.C. Calderón-Moreno, A Seidel-Walsh theorem with linear differential operators, Archiv. Math. 72 (1999), 367-375.

[3] L. Bernal-González and A. Montes-Rodríguez, Universal functions for composition operators, Complex Variables 27 (1995), 47-56.

[4] C.D. Birkhoff, Démonstration d'un théorème élémentaire sur les fonctions entières, C. R. Acad. Sci. Paris 189 (1929), 473-475.

[5] P.L. Duren, Theory of $H^{p}$ spaces, Academic Press, New York, 1970.

[6] J.B. Garnett, Bounded Analytic Functions, Academic Press, New York, 1981.

[7] P. Gorkin and R. Mortini, Universal Blaschke products, Math. Proc. Camb. Phil. Soc. 136 (2004), 175-184.

[8] K.-G. Grosse-Erdmann, Holomorphe Monster und universelle Funktionen, Mitt. Math. Sem. Giessen 176 (1987).

[9] K.-G. Grosse-Erdmann, Universal families and hypercyclic operators, Bull. Amer. Math. Soc. 36 (1999), 345-381.

[10] K.-G. Grosse-Erdmann, Recent developments in hypercyclicity, Rev. R. Acad. Cien. Serie A. Mat. 97 (2003), 273-286.

[11] G. Herzog, On a theorem of Seidel-Walsh, Periodica Math. Hungar. 30 (1995), 205-210.

[12] K. Hoffman, Banach spaces of analytic functions, Dover, New York, 1988. 
[13] U. Klein, Hardy spaces of higher order with special consideration of $H_{1}^{1}$, Travaux mathématiques VI, Luxembourg, Centre universitaire de Luxembourg, Dep. des Sciences (1994), 1-100.

[14] W.P. Seidel and J.L. Walsh, On approximation by Euclidean and non-Euclidean translates of an analytic function, Bull. Amer. Mat. Soc. 47 (1941), 916-920.

[15] K. Zhu, Operator Theory in Function Spaces, Marcel Dekker, New York, 1990.

L. BERNAL-GONZÁLEZ and M.C. CALDERÓN-MORENO DEPARTAMENTO DE ANÁLISIS MATEMÁTICO

FACULTAD DE MATEMÁTICAS, APDO. 1160

AVENIDA REINA MERCEDES

41080 SEVILLA, SPAIN

E-mails: lbernal@us.es, mccm@us.es
ANTONIO BONILLA DEPARTAMENTO DE ANÁLISIS MATEMÁTICO UNIVERSIDAD DE LA LAGUNA C/ASTROFÍSICO FCO. SÁNCHEZ, S/N 38271 LA LAGUNA, SPAIN E-mail: abonilla@ull.es 